\title{
Enhanced Four-Wave Mixing in Doubly Resonant Si Nanoresonators
}

Rémi Colom, ${ }^{\dagger, \ddagger}$ Lei Xu, "Loris Marini, ${ }^{\ddagger, \S}$ Frédéric Bedu, $\|$ Igor Ozerov, $\|$ Thomas Begou, ${ }^{\dagger}$ Julien Lumeau, ${ }^{\dagger}$ Andrey Miroshnishenko, ${ }^{\llbracket}$ Dragomir Neshev,${ }^{\perp}$ Boris

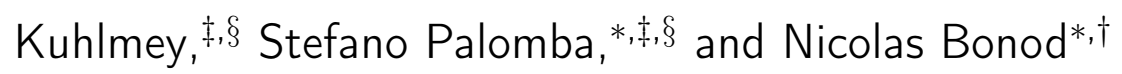

$\dagger$ CNRS, Aix-Marseille Univ, Centrale Marseille, Institut Fresnel, 13397 Marseille, France $\ddagger$ Institute of Photonics and Optical Science (IPOS), School of Physics, University of Sydney, NSW 2006, Australia

ISchool of Engineering and Information Technology, University of New South Wales, Canberra, ACT 2600, Australia

$\S$ The University of Sydney Nano Institute, University of Sydney, NSW 2006, Australia \|CNRS, Aix-Marseille Univ, CINAM, 13288 Marseille, France

$\perp$ Nonlinear Physics Centre, Research School of Physics and Engineering, The Australian National University, Canberra, ACT 2601, Australia

E-mail: stefano.palomba@sydney.edu.au; nicolas.bonod@fresnel.fr

\begin{abstract}
Frequency conversion is one of the main applications of nonlinear optical processes in which a signal is produced at a different wavelength from the excitation wavelength. In particular, four-wave mixing (FWM) is a third order non-linear optical process that allows, for instance, the generation of visible frequencies by tuning near-infrared laser pumps. Here, in order to augment the very weak FWM conversion efficiency, we design silicon Mie resonators that exhibit two resonances of the internal electric field intensity
\end{abstract}


around the frequency range of the laser pumps. The linear extinction spectrum of the individual Si resonator is first measured by bright field spectroscopy and compared with numerical simulations to confirm the existence of the two resonances corresponding to electric and magnetic dipoles excitations. The FWM signal is then measured for a single Si nanoresonator when the first pump is set to the electric resonance, while tuning the frequency of the second pump across the magnetic dipolar resonance. We show that the FWM signal generated in the visible spectrum is maximum when the frequency of the tunable pump corresponds to the maximum of the internal electric field intensity. At this position, the FWM signal is enhanced by more than two orders of magnitude compared with the FWM signal generated by the unpatterned silicon film.

\section{Introduction}

Silicon is an ubiquitous material in opto-electronics with applications in image sensors or photovoltaics. However, the spectral range of applications of silicon is limited in the visible spectrum since its band gap is located near $1.1 \mathrm{eV}$ for crystalline Si. As a consequence, silicon is transparent in the near infrared, and behaves as a dielectric characterized by a high refractive index $\approx 3.4$. Making silicon sensitive to near-infrared (NIR) radiation is very challenging and multiphoton absorption is a promising way to address this challenge. Four wave mixing $(\mathrm{FWM})$ is a third order nonlinear effect where four photons of different frequencies are mixed together and, for instance, light in the NIR can generate visible (VIS) radiation. ${ }^{1-5}$ Such nonlinear effects are generally weak since the nonlinear susceptibility $\chi^{(3)}$ is small. However, given that the nonlinear field is proportional to the product between the nonlinear susceptibility of the nonlinear medium and the applied electric field, the nonlinear field can be enhanced near the resonances. ${ }^{6}$ For this purpose, optical cavities can be designed to trap light inside the nonlinear material. In particular, high refractive index subwavelength-sized particles can host low order Mie resonances that are associated with strong electric field intensities inside or in the vicinity of the particle. ${ }^{7-16}$ Silicon nanoparticles behave as optical 
antennas able to efficiently couple the far field radiation to the near field, with applications in fluorescence or in biosensing. ${ }^{17-24}$ The possibility to engineer the near-field intensity via optical antennae has opened a novel route to develop nonlinear optics with nanostructured semi-conductors. ${ }^{25,26}$ The first demonstrations were with Mie resonators designed in group IV semiconductors (silicon, germanium) yielding third harmonic generation (THG). ${ }^{27-32}$ Alldielectric nanostructures made of crystalline silicon ${ }^{31}$ or III-V semiconductors ${ }^{33-36}$ can also be engineered to enhance the process of second harmonic generation (SHG) due to their non-centrosymmetric crystalline structure. III-V semiconductors feature excellent nonlinear properties and the coupling between their intrinsic nonlinearity with internal field enhancements in Mie resonators has strong potential for the development of highly efficient nonlinear all-dielectric metasurfaces. Besides SHG and THG, four-wave mixing (FWM) is a versatile nonlinear process that allows to tune the frequency of the nonlinear signal by modifying the frequency of one pump. ${ }^{37,38}$ In the context of Mie resonators, FWM allows for spectrally tuning the pump around the frequency of a Mie resonance in order to maximize the nonlinearity enhancement by compensating for material inhomogeneities and fabrication inaccuracies.

Here we generate visible photons by degenerate FWM when two NIR pumps are exciting an individual silicon nanodisk (Fig. 1a,b). The first pump is delivered by a tunable Ti-Sapphire laser (Coherent Chameleon Ultra) generating 140 fs pulses with a central wavelength ranging between $750 \mathrm{~nm}$ and $880 \mathrm{~nm}$. The second pump is obtained by an Optical Parametric Oscillator (OPO) (Coherent Chameleon) coupled to the main oscillator (Chameleon Ultra), as shown in Fig. 1c. The mean wavelength of the second pump can also be tuned between $1050 \mathrm{~nm}$ and $1500 \mathrm{~nm}$. Since the two femtosecond laser pulses have to overlap both in space and in time, the arrival time of the first beam with frequency $\omega_{1}$ is tuned by an optical delay line whereas the spatial overlap is controlled by positioning the point spread functions of the two pumps at the image plane of a home-built confocal microscope by a $100 \times$ and high numerical aperture $(\mathrm{NA}=0.85)$ air microscope objective (Olympus LCPLN100XIR). The visible nonlinearly generated photons from individual resonators are 
collected by the same objective and analyzed by an optical spectrometer (Princeton Instruments, Isoplane 320) coupled to a visible CCD camera (Princeton Instruments, Pixis 1024) (Fig. 1a).

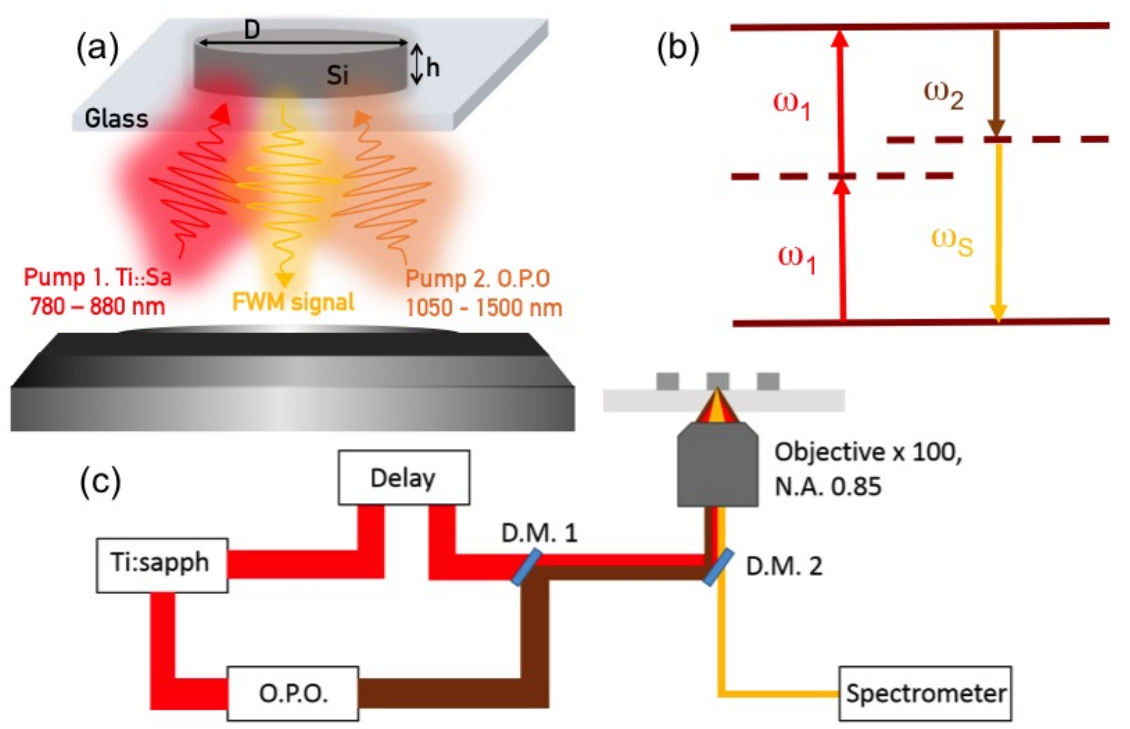

Figure 1: (a) Schematic representation of the degenerate four-wave mixing (FWM) experiment: two laser pumps of frequency $\omega_{1}$ and $\omega_{2}$ are mixed in a silicon nanodisk to generate a FWM signal at frequency $\omega_{s}=2 \omega_{1}-\omega_{2}$. (b) FWM principle. (c) Schematic representation of the experimental set-up: the laser pump beam $\omega_{1}$ is in red and $\omega_{2}$ is in brown. The FWM beam is in yellow. D. M.: Dichroic Mirror.

A schematic representation of the FWM process used is depicted in Fig. 1b. Degenerate FWM is a third order nonlinear process in which a signal at the frequency $\omega_{s}=2 \omega_{1}-\omega_{2}$ is generated when the sample is illuminated by the two pumps at frequencies $\omega_{1}$ and $\omega_{2}$. Assuming that the material we consider is amorphous (this assumption shall be discussed hereafter), the non-linear polarization associated with this process has the following form:

$$
\mathbf{P}_{N L, \omega_{s}}(\mathbf{r})=6 \epsilon_{0} \chi_{1122}^{(3)}\left(\mathbf{E}_{\omega_{1}}(\mathbf{r}) \cdot \overline{\mathbf{E}}_{\omega_{2}}(\mathbf{r})\right) \mathbf{E}_{\omega_{1}}(\mathbf{r})+3 \epsilon_{0} \chi_{1221}^{(3)}\left(\mathbf{E}_{\omega_{1}}(\mathbf{r}) \cdot \mathbf{E}_{\omega_{1}}(\mathbf{r})\right) \overline{\mathbf{E}}_{\omega_{2}}(\mathbf{r})
$$

where $\chi_{1122}^{(3)}$ and $\chi_{1221}^{(3)}$ are two elements of the third-order susceptibility tensor of amorphous silicon and the nonlinear signal has a frequency $\omega_{s}=2 \omega_{1}-\omega_{2}$. 


\section{Results and discussion}

Silicon nanodisks are designed to exhibit two resonances yielding internal field maxima near the wavelengths of the two chosen pumps $\omega_{1} \approx 850 \mathrm{~nm}$ and $\omega_{2} \approx 1200 \mathrm{~nm}$. The nanodisks are fabricated by nanopatterning of a thin film of amorphous silicon. However, illumination of amorphous silicon by femto-second laser pulses leads to a crystallization of silicon as will be discussed later. ${ }^{39,40}$ The actual FWM measurements will then be performed after

the crystallization of silicon. Subsequently, in our simulations we use the dispersion data of crystalline silicon for calculating the optical response of the silicon nanodisks on a glass substrate.

Let us consider the average of the internal field enhancement $\mathbf{E}_{\text {int }} / \mathbf{E}_{0}$ over the whole volume of the silicon disk:

$$
\left\langle\left|\mathbf{E}_{\text {int }}\right|\right\rangle=\frac{\int_{V_{D}}\left|\mathbf{E}_{\text {int }}\right| d \mathbf{r}}{\int_{V_{D}}\left|\mathbf{E}_{0}\right| d \mathbf{r}},
$$

where $V_{D}$ is the volume of the scatterer while $\mathbf{E}_{0}$ is the amplitude of the excitation field. The internal field enhancement and its average in the particle $\left\langle\left|\mathbf{E}_{\text {int }}\right|\right\rangle$ are calculated by Finite Element Methods (FEM) (COMSOL Multiphysics). We found that a silicon cylinder with a diameter $D=340 \mathrm{~nm}$ and a height $h=240 \mathrm{~nm}$ on a glass substrate, exhibits two resonances of the internal field $\left\langle\left|\mathbf{E}_{\text {int }}\right|\right\rangle$ at $\omega_{1}$ and $\omega_{2}$, for which the spectrum is plotted in Fig. 2a. The two maxima in the spectral field distribution are located in the NIR. The nature of these two resonances can be determined by computing a multipolar decomposition of the optical response of the nanodisk. In Fig. 2c, the extinction cross-section of the nanodisk ( $D=340 \mathrm{~nm}$ and a height $h=240 \mathrm{~nm})$ and its multipolar expansion are plotted as a function of the wavelength.

The spectral peak at $\lambda \approx 1205 \mathrm{~nm}$ is associated with a magnetic-dipolar resonance. This multipolar analysis is confirmed by observing that the calculated field distribution at $\lambda=1205 \mathrm{~nm}$ in Fig. $2 \mathrm{~d}$ is similar to a magnetic dipole field distribution. A counterpart to the second peak of the internal field average can also be found in the extinction spectrum located 
at $\lambda \approx 840 \mathrm{~nm}$. The multipolar analysis of this second peak reveals that it is mostly due to an electric dipolar response combined with a weaker magnetic quadrupolar contribution. Two peaks of the ED can be observed in the spectrum near $\lambda \approx 840 \mathrm{~nm}$ and $\lambda \approx 1050 \mathrm{~nm}$ that are associated with very different electric field distributions inside the nanoresonator. Different from the resonant peak at $\lambda \approx 1050 \mathrm{~nm}$ where the electric field is mostly enhanced around the surface of the nano-resonators, the electric field for the peak at $\lambda \approx 840 \mathrm{~nm}$, penetrates inside the nano-resonator, resulting in a strong internal field enhancement which can further facilitate light-matter interactions and augment the nonlinear process.

A careful analysis of Eq. 1 shows that the nonlinear polarization depends on two terms $\left(\mathbf{E}_{\omega_{1}}(\mathbf{r}) \cdot \overline{\mathbf{E}}_{\omega_{2}}(\mathbf{r}) \mathbf{E}_{\omega_{1}}(\mathbf{r})\right)$ and $\left(\mathbf{E}_{\omega_{1}}(\mathbf{r}) \cdot \mathbf{E}_{\omega_{1}}(\mathbf{r})\right) \overline{\mathbf{E}}_{\omega_{2}}(\mathbf{r})$. We therefore plot in Fig. 2b the overlap integrals:

$$
\begin{aligned}
I_{N L_{1}} & =\left|\iiint_{V_{D}}\left(\mathbf{E}_{\omega_{1}}(\mathbf{r}) \cdot \overline{\mathbf{E}}_{\omega_{2}}(\mathbf{r})\right) \mathbf{E}_{\omega_{1}}(\mathbf{r}) d^{3} \mathbf{r}\right|, \\
I_{N L_{2}} & =\left|\iiint_{V_{D}}\left(\mathbf{E}_{\omega_{1}}(\mathbf{r}) \cdot \mathbf{E}_{\omega_{1}}(\mathbf{r})\right) \overline{\mathbf{E}}_{\omega_{2}}(\mathbf{r}) d^{3} \mathbf{r}\right| .
\end{aligned}
$$

The color maps in Fig. 2b provide information on the FWM generation with respect to the $\omega_{1}$ and $\omega_{2}$ frequencies showing four areas in which the FWM signal is enhanced. The peak that occurs in the area corresponding to the spectral range of the two pumps, i.e. $\lambda_{1}=[800-900] \mathrm{nm}$ and $\lambda_{2}=[1150-1300] \mathrm{nm}$, is more pronounced $I_{N L_{2}}$. As the FWM signal occurs at the wavelength $\lambda_{s}=\frac{\lambda_{1} \lambda_{2}}{2 \lambda_{2}-\lambda_{1}}$, the peak observed on the top left of the $I_{N L 1}$ and $I_{N L 2}$ color maps, i.e. for $\lambda_{1} \approx 840 \mathrm{~nm}$ and $\lambda_{2} \approx[1200-1300] \mathrm{nm}$, will yield a FWM signal in the visible at $\lambda_{s} \approx[620-646] \mathrm{nm}$ for $\lambda_{1} \approx 840 \mathrm{~nm}$. The femtosecond pumps induce a crystallization of silicon after illumination of the nanodisk. The FWM signal in monocrystalline silicon should be rigorously modeled by three terms ${ }^{42}$ while Eq. (1) that includes two terms is valid in amorphous silicon. However, the femtosecond pumps induce a polycrystallization of the silicon nanodisk with a random orientation of the crystalline meshes. The non-linear tensor used when modeling the FWM signal in our experiment can 
(a)

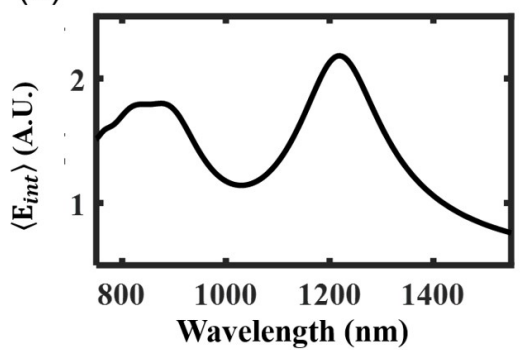

(c)

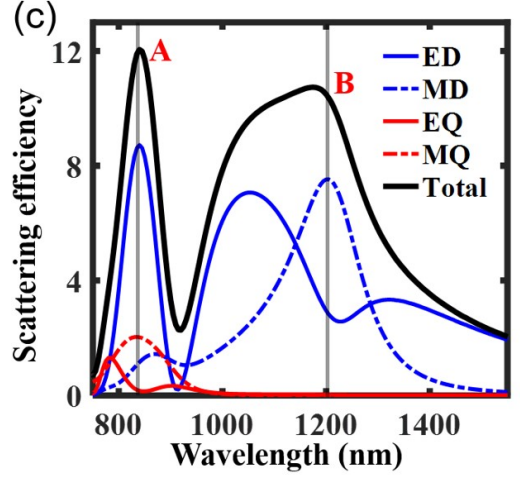

(b)
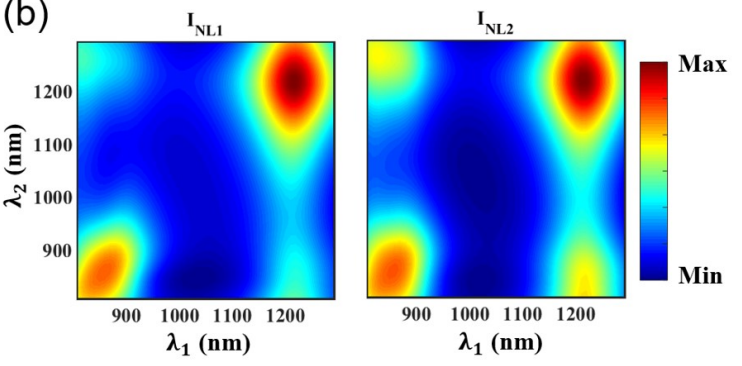

(d)

A:
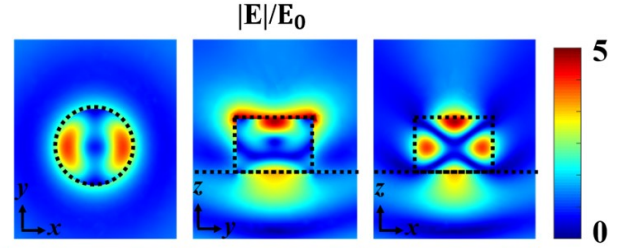

B:
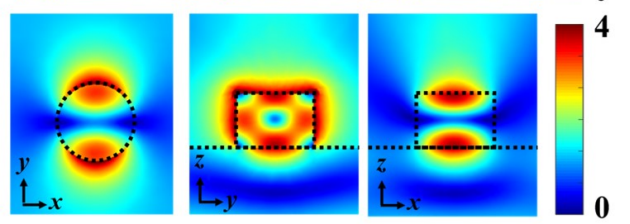

Figure 2: (a) Spectrum in the near-infrared of the simulated internal field enhancement averaged in the whole volume of the crystalline silicon disk, ${ }^{41}\left\langle\left|\mathbf{E}_{\text {int }}\right|\right\rangle$, chosen to feature two resonances at $\lambda_{1}$ and $\lambda_{2}$ : diameter $D=340 \mathrm{~nm}$ and height $h=240 \mathrm{~nm}$. (b) Overlap integrals $I_{N L 1}$ (left) and $I_{N L 2}$ (right) calculated with Eqs. 3-4 and plotted with respect to $\lambda_{1}$ and $\lambda_{2}$. (c) Scattering spectrum and multipolar decomposition calculated for the chosen $\mathrm{Si}$ particle $(D=340 \mathrm{~nm}, h=240 \mathrm{~nm})$ on a substrate of refractive index 1.5. Full calculation: black curve; colored curves: multipolar decomposition. Blue curves: electric (full line) and magnetic (dotted line) dipolar contribution. Red curves: electric (full line) and magnetic (dotted line) quadrupolar contribution. (d) Electric near-field distributions for wavelength position $\mathrm{A}$ and $\mathrm{B}$ as depicted in (c) corresponding to the excitation of $2^{\text {nd }} \mathrm{ED}$ and $1^{\text {st }}$ MD resonances, respectively. All calculations are carried out with the permittivity data of crystalline silicon. ${ }^{41}$

therefore be assumed to have the same symmetry as in amorphous silicon and accurately modeled with Eq. (1).

Before investigating the nonlinear optical properties of the fabricated silicon Mie resonators, we verified that the geometrical and optical properties of the samples are in agreement with the expected morphology and optical behaviour in the linear regime. The former is first investigated by a scanning electron beam microscope and the diameter of the disk is found in reasonable agreement with the targeted value $D=340 \mathrm{~nm}$ (see Fig. 3a). Second, we 

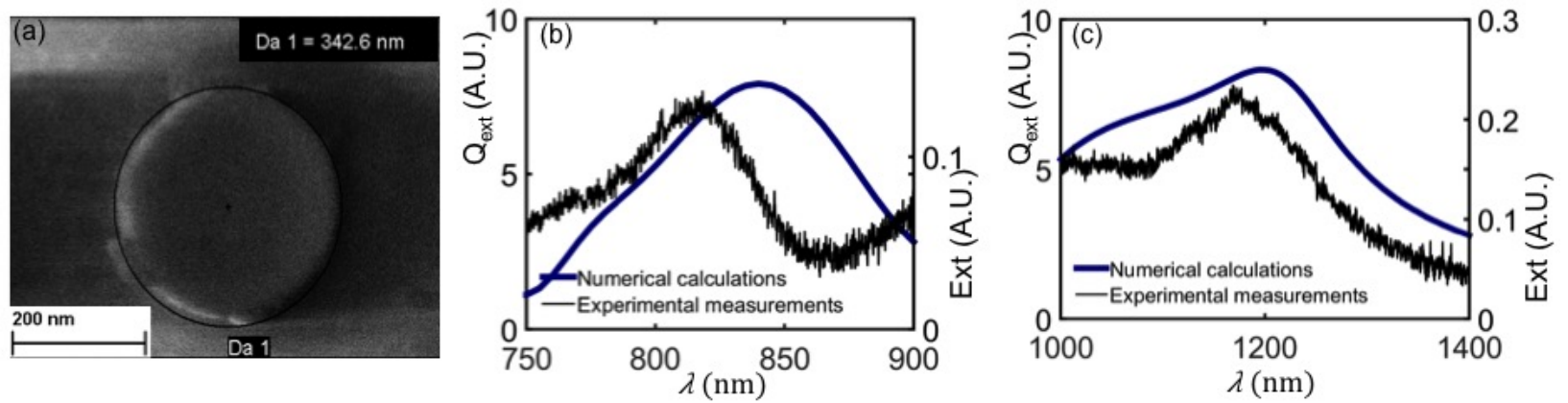

Figure 3: (a) Image of a fabricated Si resonator obtained by Scanning Electron Microscopy (SEM). The measurement of the diameter of the Si nanodisk gives a diameter equal to $342 \mathrm{~nm}$. (b,c) Comparison between the measured and the calculated extinction spectra in the visible (b) and near infrared (c) spectrum. Full black line, left scale: experimental measurements of the extinction spectrum; blue full line, right scale: numerical calculations of the extinction cross-section of a silicon cylinder of diameter $D=340 \mathrm{~nm}$ and height $h=240 \mathrm{~nm}$ with crystalline silicon. ${ }^{41}$

verified that the linear response of isolated nanodisks before the crystallization of silicon, i.e. before illuminating the silicon nanodisk with the femtosecond pumps, matches the numerical prediction of the extinction cross-section calculated with amorphous silicon (See SI).

Since the generation of a FWM signal requires an excitation of the sample by two highpower femtosecond laser pumps, a modification of the extinction spectrum is observed after the first illumination by the pumps. This change corresponds to a blue-shift of the resonance wavelengths in the extinction spectrum. This blue shift of the spectra is assumed to result from the crystallization of the initially amorphous silicon nanodisks due to the pulsed laser illumination ${ }^{39,43}$ and is confirmed by numerical calculations of the extinction spectra performed with the refractive index of crystalline silicon (See SI). We verified that after the crystallization induced by the first illumination from the two laser pumps, the resonances do not exhibit any further spectral shift. Therefore, we show in Fig. 3b,c the stable spectra obtained after several illuminations (spectra acquired in the visible and NIR are obtained with two different cameras which explains why they are plotted in two different figures). The noise observed in Fig. 3b,c comes from the fact that extinction spectra are measured at the 
single resonator scale. Hence, the FWM measurements are performed only after illuminating the nanoresonators and verifying that crystallization has occurred which stabilizes the spectral position of the resonances.

The intensity of the FWM signal is measured with the camera while sweeping the wavelength $\lambda_{2}$ of the second pump across the NIR resonance of the nanoresonator after crystallization has occurred. The first pump is set at wavelength $\lambda_{1}=810 \mathrm{~nm}$, matching the high frequency electric dipolar resonance of the Si disk.

In order to assure that the pump excitation has not further modified the properties of the silicon resonator, the extinction of the silicon nanodisk is measured before and after each excitation of the FWM signal. The FWM signal enhancement yielded by the individual silicon resonator is compared with the FWM signal measured from an unpatterned silicon thin film. This measurement of the FWM signal on the pristine silicon film is used as a reference. In addition to this reference, all measurements are normalized by the intensity of the two pumps (normalization of the FWM signal is detailed in Methods). The FWM spectra obtained from the pristine thin film and from the silicon Mie resonator are displayed in Fig. 4 for six different wavelengths $\lambda_{2}$ of the pump 2. The FMW signal generated by individual nanoresonators is more than two-orders of magnitude higher than the one measured from the unpatterned Si film (Fig. 4a,b).

The spectral dependence of the FWM signal generated by the silicon film may be due to several factors. First, the third-order susceptibility is certainly dispersive. This makes the nonlinear response of the thin film dependent both on the wavelengths of the pumps and on the wavelength of the signal. Second, the dependence of the FWM signal may also be caused by the excitation of Fabry-Perot resonances inside the silicon slab. Regarding the FWM response of the silicon nanodisk, it has a maximum at $\lambda_{2}=1200 \mathrm{~nm}$ and decreases for shorter and larger values of $\lambda_{2}$. The significance of the Mie resonances for enhancing the FWM signal is clear when observing the two spectra in Fig. 4a,b since the FWM signal generated by the silicon particles can be two-orders of magnitude higher than that measured 
(a)
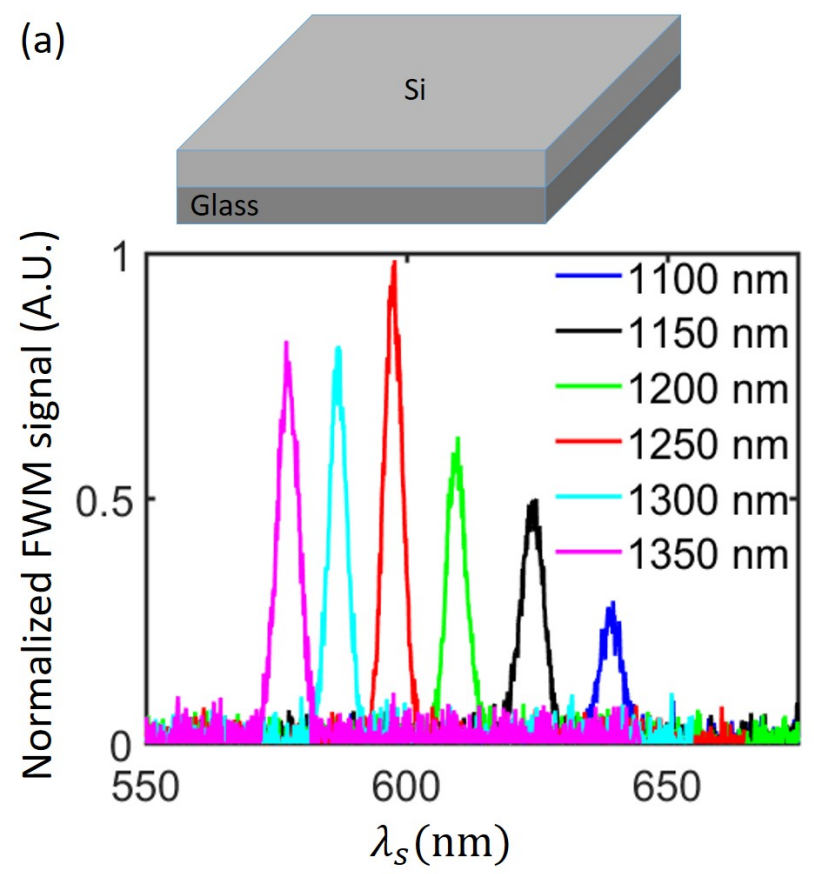

(b)
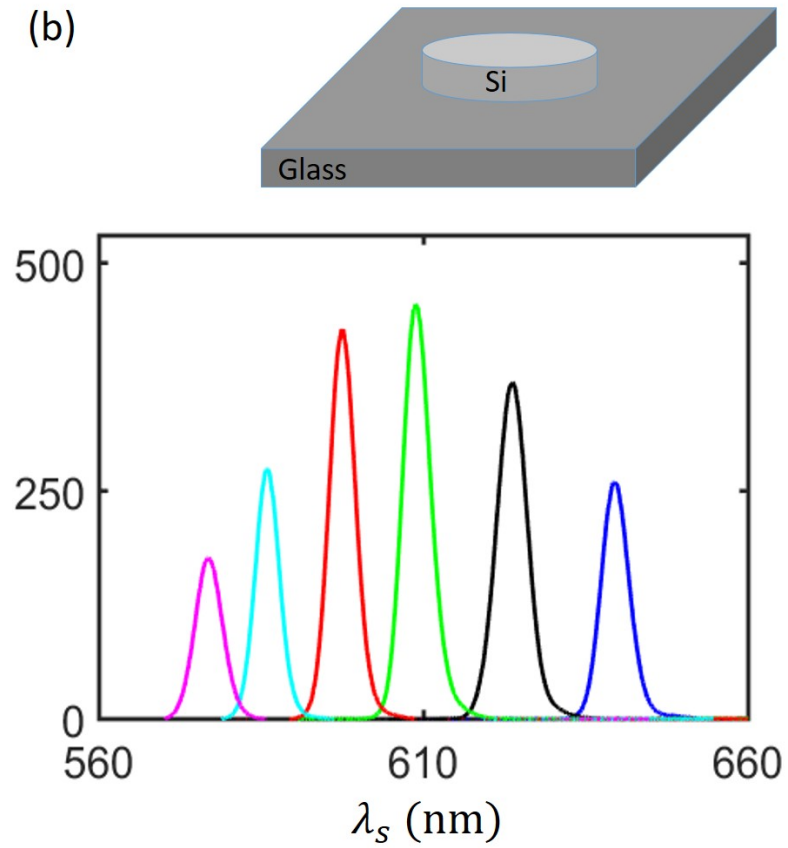

Figure 4: Comparison of the normalized FWM signal measurement on (a) an unpatterned silicon layer and (b) on a silicon nanodisk of diameter $D=340 \mathrm{~nm}$ and height $h=240 \mathrm{~nm}$ when the wavelength of the second pump $\lambda_{2}$ is changed. The values in the legend are the different values of $\lambda_{2}$

on the unpatterned Si film.

In order to better understand the enhancement of the FWM intensity, we plot in Fig. 5 the FWM signal intensity as a function of the wavelength $\lambda_{2}$ of the second pump. This signal intensity is compared to the square of the internal field enhancement defined in Eq. (2) calculated with the dielectric permittivity of crystalline silicon in Fig. $5 .{ }^{41} \mathrm{~A}$ good agreement is found between the internal field enhancement and the FWM signal. This is in agreement with the fact that a FWM signal is proportional to $\propto\left|\left(\mathbf{E}_{\omega_{1}}(\mathbf{r}) \cdot \mathbf{E}_{\omega_{1}}(\mathbf{r})\right) \mathbf{E}_{\omega_{2}}(\mathbf{r})\right|^{2}$. This explains why the two fold enhancement of the internal field intensity $\left\langle\left|\mathbf{E}_{\text {int }}\right|\right\rangle^{2}$ induces a nearly two fold enhancement of the FWM signal. Let us stress that we tuned only $\lambda_{2}$ while the first pump matches the electric dipole resonance. This explains why the gain efficiency brought by the magnetic resonance is limited by 2 , but that combining the two resonances leads to a FWM signal enhancement of two orders of magnitude compared with the thin 

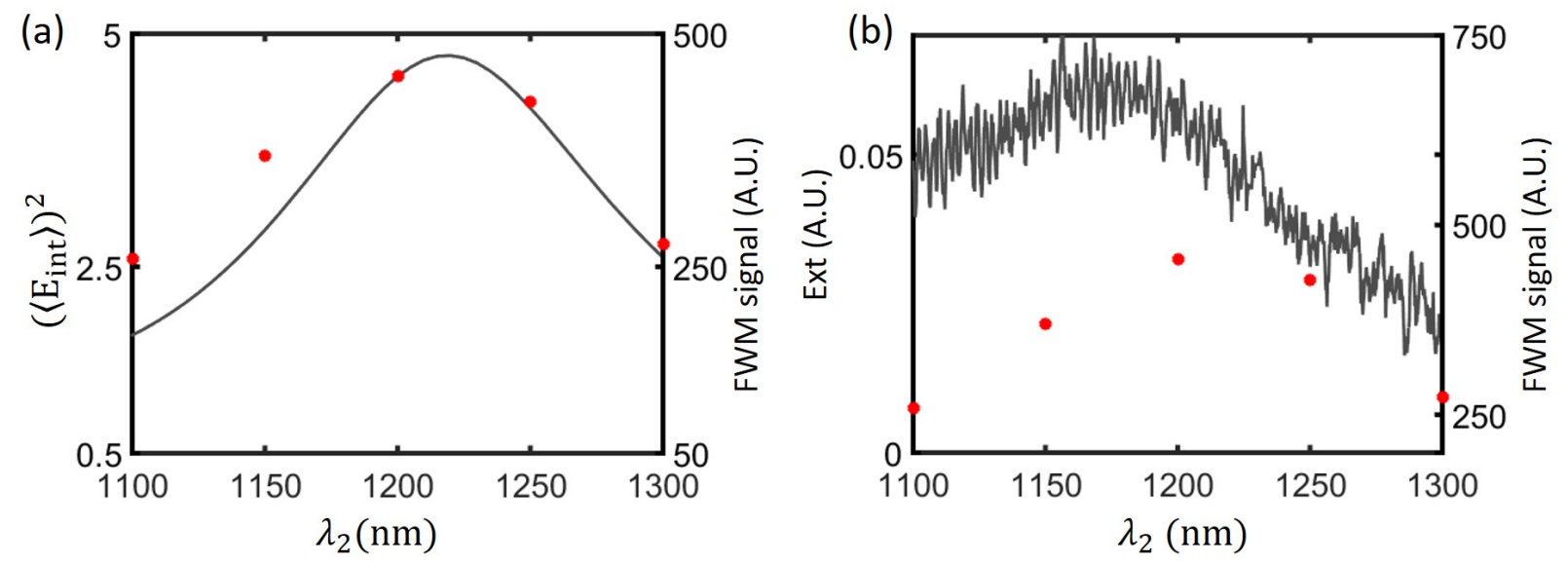

Figure 5: FWM signal normalized by the pump intensities plotted in red dots with respect to the wavelength $\lambda_{2}$ of pump 2 and compared with (a) the spectrum of the internal field enhancement $\left(\left\langle\left|\mathbf{E}_{\text {int }}\right|\right\rangle\right)^{2}$ calculated with Eq. (2) and with (b) the measured extinction crosssection calculated with the permittivity of crystalline silicon.

film.

In Fig. 5(b), we plot the FWM signal and the extinction measurement on the same figure in order to compare the peak of the FWM signal to the resonance of the nanodisk extinction. There appears indeed to be a red-shift of the FWM peak with respect to the resonance of the extinction of the crystallized nanodisk. This red-shift is expected since the resonance of the internal field is classically red-shifted compared to the resonance of the extinction cross section. ${ }^{14}$ The stronger extinction cross-section observed in Fig. 5(b) at shortest wavelengths, around $1100 \mathrm{~nm}$, may be due to a larger scattering of light caused by the resonant excitation of an electric dipole inside the Si particle. This electric dipole is associated with a strong enhancement of light intensity around the surface of the particle, as displayed in Fig.2d, which is expected to increase the sensitivity to surface scattering.

\section{Conclusion}

In conclusion, we combined linear and non-linear spectroscopy to report the generation of visible light by exciting a single silicon particle in the NIR spectrum with two pumps. This particle was designed to exhibit two different resonances, namely an electric dipolar 
resonance associated a magnetic quadrupolar resonance excited by the first pump and a magnetic dipolar resonance FWM experiments with silicon. Higher order resonances could also certainly yield strong FWM signal enhancements, but at the cost of a much larger fingerprint of the photonic nanostructure. The frequency of one pump was swept over the magnetic resonance to verify that the two fold enhancement of the internal field in the volume of the Si particle yielded an almost two fold enhancement of the FWM intensity. This experiment was performed while keeping the frequency of the other pump at the electric dipole frequency. The excitation of the two different resonances leads to an enhancement of the FWM intensity in the visible spectrum of two orders of magnitude compared with

a thin Si film of same thickness. Our study proves the importance of internal electric field enhancement yielded by Mie resonances to enhance the frequency conversion efficiency from the near-infrared to the visible spectrum. All-dielectric nanophotonics therefore appears as a promising route for upconverting near infrared signals to higher frequencies and for detecting infrared photons with indirect bandgap semiconductors.

\section{Methods}

\section{Nanodisk fabrication}

Silicon nanodisks are fabricated by e-beam lithography (EBL) along with reactive-ion etching. A $240 \mathrm{~nm}$ thick layer of amorphous silicon is deposited onto a $150 \mu \mathrm{m}$ thick glass substrate by plasma assisted reactive magnetron sputtering (Buhler, HELIOS). A 60 nm thick layer of a PMMA (poly(methyl-methacrylate)) positive e-beam resist is spin-coated on top of the silicon film. Arrays with a $10 \mu \mathrm{m}$ pitch of nanocircles with diameter ranging from $250 \mathrm{~nm}$ to $360 \mathrm{~nm}$ are then patterned by e-beam lithography (Pioneer, Raith). The sample is then developed in a commercial MIBK-isopropanol solution. A layer of nickel, $16 \mathrm{~nm}$ in thickness, is then evaporated on top of the sample and acts as a hard mask during the reactive-ion etching step. After the resist development, the remaining PMMA and 
the nickel layer on top of it are removed in a lift-off process by putting the samples into a ultrasonic bath of ethyl lactate. After this step, the nickel mask remains only on the nanodisks patterned during the e-beam lithography step. The zones of the silicon layer that are not covered by the nickel mask are then etched by reactive-ion etching (MG-200, Plassys) by a gas mixture containing $\mathrm{SF}_{6}, \mathrm{O}_{2}$ and $\mathrm{CHF}_{3}$ and with a pure $\mathrm{O}_{2}$ plasma. Finally, the remaining nickel mask is removed in an acid solution of $\mathrm{HCl}$ and $\mathrm{FeCl}_{3}$.

\section{Linear characterization}

For the linear characterization of the isolated silicon nanodisks, the extinction spectrum is measured with a confocal microscope. The sample is illuminated by a collimated white source slightly focused in order to combine intensity and homogeneity of the beam on the sample. The light from the sample is subsequently collected by a $100 \times$ magnifying objective with a numerical aperture equal to 0.85 (Olympus LCPLN100XIR) and then focused on the slit of the photospectrometer (Princeton Instrument, Isoplane 320) that acts as a spatial filter. In fact, only the light coming from the image of the slit onto the sample is collected by the photospectrometer. Light scattered by an isolated Si nanodisk is then collected by adequately closing the slit to spatially filter the light signal in one direction and by using the binning option of the camera to spatially filter in the other direction. Extinction measurements are then performed by measuring the light spectrum transmitted through (i) an isolated disk $I_{d}$ and (ii) the substrate $I_{s}$ and the background noise $I_{b}$. Extinction spectrum is defined by $\operatorname{Ext}(\lambda)=-\frac{I_{d}(\lambda)-I_{s}(\lambda)}{I_{s}(\lambda)-I_{b}(\lambda)}$.

\section{Nonlinear characterization}

Four-wave mixing measurements are performed using the experimental setup displayed in Fig. 1. The first pump is a femtosecond pulse centered at the wavelength $\lambda_{1}$ generated by a Ti-sapphire laser (Coherent Chameleon Ultra). The second pump is a femtosecond pulse centered at the wavelength $\lambda_{2}$, produced by an optical parametric oscillator (OPO) pumped 
by the Ti-sapphire laser. The output beams from the Ti:sapphire laser and the OPO are then sent to the microscope in two different optical paths, one of which passes through an optical delay line for controlling the temporal overlap of the pump pulses.

The two linearly polarized beams are merged before entering in an optical microscope via a dichroic mirror (Thorlabs, DMSP1000R). The laser pulses are then reflected by another dichroic mirror (cutoff wavelength $805 \mathrm{~nm}$, Thorlabs DMSP805R) and then focused onto the sample by a $100 \times, 0.85$ numerical aperture objective (Olympus LCPLN100XIR). A perfect spatial overlap is necessary to produce a FWM optical signal at the image plane of the objective where the sample is located. The temporal overlap of the pulses generated by each source is obtained by adjusting the optical delay line until the FWM signal is maximized. The reflected FWM signal is then collected by the same objective and subsequently sent to a spectrometer.

We measure the FWM signal from the Si nanoresonators and comparing it to the FWM signal obtained from a Si thin-film. The row data from the spectrometer are normalized in order to take into account the change of average intensity on the sample when the wavelength of the pumps is changed. For that purpose, we measured the average powers of the two pumps and normalized them by the surface of the focused spot. This area was estimated by using the radius of a diffraction limited system: $r(\lambda)=0.61 \frac{\lambda}{N A}$ where $N A$ is the numerical aperture of the objective that in our case is equal to $N A=0.85$. The normalization factor

for the FWM measurements is consequently equal to $N_{\text {norm }}=\left(\frac{P_{\lambda_{1}}}{\pi r\left(\lambda_{1}\right)^{2}}\right)^{2} \frac{P_{\lambda_{2}}}{\pi r\left(\lambda_{2}\right)^{2}}$. Finally the signal from the silicon nanodisks and the FWM signal from the layer are both normalized by the maximum FWM intensity from the silicon layer: $3.5 * 10^{22}$.

\section{Numerical calculation method}

For the numerical simulation of linear scattering, we use a finite-element-method solver in the frequency domain from COMSOL Multiphysics to model the linear response from our $\mathrm{Si}$ resonator. As the presence of substrate makes it difficult to perform the multipolar analysis 
using the scattered fields directly, the multipolar decomposition analysis here is performed using the polarization currents induced inside the Si resonator. ${ }^{44}$ By considering the fields radiated into air, we calculate and predict the contributions associated with each multipole from our Si resonator.

\section{Acknowledgement}

Research conducted within the context of the International Associated Laboratory ALPhFA: Associated Laboratory for Photonics between France and Australia. This work has been carried out thanks to the support of the A*MIDEX project (no. ANR-11-IDEX-0001-02) funded by the Investissements d'Avenir French Government program, managed by the French National Research Agency (ANR). We acknowledge support by the Australian Research Council. Nanofabrication processes were performed in PLANETE CT PACA cleanroom facility (CINaM, Marseille). The authors thank Prof. Yuri Kivshar and Dr Brian Stout for fruitful discussions and valuable comments.

\section{References}

(1) Danckwerts, M.; Novotny, L. Physical Review Letters 2007, 98, 026104.

(2) Palomba, S.; Novotny, L. Physical Review Letters 2008, 101, 056802.

(3) Renger, J.; Quidant, R.; Van Hulst, N.; Novotny, L. Physical Review Letters 2010, 104, 046803.

(4) Duffin, T. J.; Nielsen, M. P.; Diaz, F.; Palomba, S.; Maier, S. A.; Oulton, R. F. Optics Letters 2016, 41, 155-158.

(5) Nielsen, M. P.; Shi, X.; Dichtl, P.; Maier, S. A.; Oulton, R. F. Science 2017, 358, 1179-1181. 
(6) Fix, B.; Jaeck, J.; Vest, B.; Verdun, M.; Beaudoin, G.; Sagnes, I.; Pelouard, J.-L.; Haïdar, R. Applied Physics Letters 2017, 111, 041102.

(7) García-Etxarri, A.; Gómez-Medina, R.; Froufe-Pérez, L. S.; López, C.; Chantada, L.; Scheffold, F.; Aizpurua, J.; Nieto-Vesperinas, M.; Sáenz, J. J. Optics Express 2011, 19, $4815-4826$.

(8) Kuznetsov, A. I.; Miroshnichenko, A. E.; Fu, Y. H.; Zhang, J.; Luk'yanchuk, B. Scientific Reports 2012, 2, 492.

(9) Albella, P.; Poyli, M. A.; Schmidt, M. K.; Maier, S. A.; Moreno, F.; Sáenz, J. J.; Aizpurua, J. The Journal of Physical Chemistry C 2013, 117, 13573-13584.

(10) Bakker, R. M.; Permyakov, D.; Yu, Y. F.; Markovich, D.; Paniagua-Domínguez, R.; Gonzaga, L.; Samusev, A.; Kivshar, Y.; Luk'yanchuk, B.; Kuznetsov, A. I. Nano Letters 2015, 15, 2137-2142.

(11) Decker, M.; Staude, I. Journal of Optics 2016, 18, 103001.

(12) Kuznetsov, A. I.; Miroshnichenko, A. E.; Brongersma, M. L.; Kivshar, Y. S.; Luk'yanchuk, B. Science 2016, 354, aag2472.

(13) Tribelsky, M. I.; Miroshnichenko, A. E. Physical Review A 2016, 93, 053837.

(14) Colom, R.; Devilez, A.; Bonod, N.; Stout, B. Physical Review B 2016, 93, 045427.

(15) Rybin, M. V.; Koshelev, K. L.; Sadrieva, Z. F.; Samusev, K. B.; Bogdanov, A. A.; Limonov, M. F.; Kivshar, Y. S. Physical Review Letters 2017, 119, 243901.

(16) Colom, R.; McPhedran, R.; Stout, B.; Bonod, N. Physical Review B 2018, 98, 085418.

(17) Caldarola, M.; Albella, P.; Cortés, E.; Rahmani, M.; Roschuk, T.; Grinblat, G.; Oulton, R. F.; Bragas, A. V.; Maier, S. A. Nature Communications 2015, 6, 7915. 
(18) Regmi, R.; Berthelot, J.; Winkler, P. M.; Mivelle, M.; Proust, J.; Bedu, F.; Ozerov, I.; Begou, T.; Lumeau, J.; Rigneault, H.; Garcia-Parajo, M. F.; Bidault, S.; Wenger, J.; Bonod, N. Nano Letters 2016, 16, 5143-5151.

(19) Bontempi, N.; Chong, K. E.; Orton, H. W.; Staude, I.; Choi, D.-Y.; Alessandri, I.; Kivshar, Y. S.; Neshev, D. N. Nanoscale 2017, 9, 4972-4980.

(20) Yavas, O.; Svedendahl, M.; Dobosz, P.; Sanz, V.; Quidant, R. Nano letters 2017, 17, $4421-4426$.

(21) Cambiasso, J.; Grinblat, G.; Li, Y.; Rakovich, A.; Cortés, E.; Maier, S. A. Nano Letters 2017, 17, 1219-1225.

(22) Krasnok, A.; Caldarola, M.; Bonod, N.; Alú, A. Advanced Optical Materials 2018, 6, 1701094 .

(23) Cambiasso, J.; König, M.; Cortès, E.; Schlücker, S.; Maier, S. A. ACS Photonics 2018, $5,1546-1557$.

(24) Tittl, A.; Leitis, A.; Liu, M.; Yesilkoy, F.; Choi, D.-Y.; Neshev, D. N.; Kivshar, Y. S.; Altug, H. Science 2018, 360, 1105-1109.

(25) Smirnova, D.; Kivshar, Y. S. Optica 2016, 3, 1241-1255.

(26) Krasnok, A.; Tymchenko, M.; Alù, A. Materials Today 2018, 21, 8-21.

(27) Shcherbakov, M. R.; Neshev, D. N.; Hopkins, B.; Shorokhov, A. S.; Staude, I.; MelikGaykazyan, E. V.; Decker, M.; Ezhov, A. A.; Miroshnichenko, A. E.; Brener, I.; Fedyanin, A. A.; Kivshar, Y. S. Nano Letters 2014, 14, 6488-6492.

(28) Yang, Y.; Wang, W.; Boulesbaa, A.; Kravchenko, I. I.; Briggs, D. P.; Puretzky, A.; Geohegan, D.; Valentine, J. Nano Letters 2015, 15, 7388-7393. 
(29) Grinblat, G.; Li, Y.; Nielsen, M. P.; Oulton, R. F.; Maier, S. A. Nano letters 2016, 16, $4635-4640$.

(30) Shorokhov, A. S.; Melik-Gaykazyan, E. V.; Smirnova, D. A.; Hopkins, B.; Chong, K. E.; Choi, D.-Y.; Shcherbakov, M. R.; Miroshnichenko, A. E.; Neshev, D. N.; Fedyanin, A. A.; Kivshar, Y. S. Nano Letters 2016, 16, 4857-4861.

(31) Melik-Gaykazyan, E. V.; Shcherbakov, M. R.; Shorokhov, A. S.; Staude, I.; Brener, I.; Neshev, D. N.; Kivshar, Y. S.; Fedyanin, A. A. Phil. Trans. R. Soc. A 2017, 375, 20160281.

(32) Xu, L.; Rahmani, M.; Zangeneh Kamali, K.; Lamprianidis, A.; Ghirardini, L.; Sautter, J.; Camacho-Morales, R.; Chen, H.; Parry, M.; Staude, I.; Zhang, G.; Neshev, D.; Miroshnichenko, A. E. Light: Sci. \& Appl. 2018, \%, 44.

(33) Carletti, L.; Locatelli, A.; Stepanenko, O.; Leo, G.; De Angelis, C. Optics Express 2015, 23, $26544-26550$.

(34) Carletti, L.; Locatelli, A.; Neshev, D.; De Angelis, C. ACS Photonics 2016, 3, 15001507.

(35) Gili, V.; Carletti, L.; Locatelli, A.; Rocco, D.; Finazzi, M.; Ghirardini, L.; Favero, I.; Gomez, C.; Lemaître, A.; Celebrano, M.; De Angelis, C.; Leo, G. Optics Express 2016, 24, 15965-15971.

(36) Ghirardini, L.; Marino, G.; Gili, V. F.; Favero, I.; Rocco, D.; Carletti, L.; Locatelli, A.; De Angelis, C.; Finazzi, M.; Celebrano, M.; Neshev, D. N.; Leo, G. Nano Letters 2018, $18,6750-6755$.

(37) Grinblat, G.; Li, Y.; Nielsen, M. P.; Oulton, R. F.; Maier, S. A. ACS Photonics 2017, 4, 2144-2149. 
(38) Liu, S.; Vabishchevich, P. P.; Vaskin, A.; Reno, J. L.; Keeler, G. A.; Sinclair, M. B.; Staude, I.; Brener, I. Nature Communications 2018, 9, 2507.

(39) Zywietz, U.; Evlyukhin, A. B.; Reinhardt, C.; Chichkov, B. N. Nature Communications 2014, 5, 3402 .

(40) Makarov, S.; Kolotova, L.; Starikov, S.; Zywietz, U.; Chichkov, B. Nanoscale 2018, 10, 11403-11409.

(41) Green, M. A. Solar Energy Materials and Solar Cells 2008, 92, 1305-1310.

(42) Lin, Q.; Painter, O. J.; Agrawal, G. P. Optics Express 2007, 15, 16604-16644.

(43) Dmitriev, P.; Makarov, S.; Milichko, V.; Mukhin, I.; Gudovskikh, A.; Sitnikova, A.; Samusev, A.; Krasnok, A.; Belov, P. Nanoscale 2016, 8, 5043-5048.

(44) Grahn, P.; Shevchenko, A.; Kaivola, M. New Journal of Physics 2012, 14, 093033. 
Graphical TOC Entry

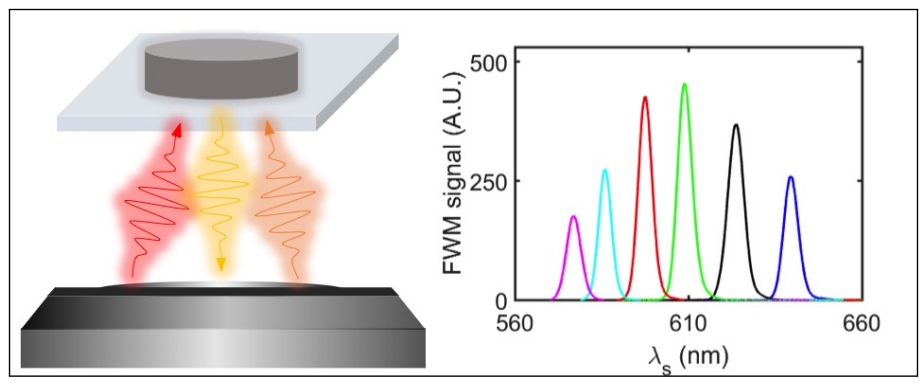

